\title{
Impact of Primary Tumor Localization on the Efficacy of Bevacizumab in Metastatic Colorectal Cancer
}

\author{
FRANK JORDAN ${ }^{1}$, NINA GRUNDMANN ${ }^{2}$, GERHARD SCHENKIRSCH ${ }^{2}$, BRUNO MÄRKL $^{3}$, \\ HELMUTH MESSMANN ${ }^{4}$, MATTHIAS ANTHUBER ${ }^{5}$, CHRISTOPH SCHMID ${ }^{1}$ and MARTIN TREPEL ${ }^{1,6}$ \\ Departments of ${ }^{1}$ Hematology and Oncology, ${ }^{3}$ Pathology, ${ }^{4}$ Gastroenterology, \\ and ${ }^{5}$ General and Visceral Surgery, and ${ }^{2}$ Central Cancer Registry, Augsburg Medical Center, Augsburg, Germany; \\ ${ }^{6}$ Department of Oncology and Hematology, University Medical Center Hamburg-Eppendorf, Hamburg, Germany
}

\begin{abstract}
Aim: Right- and left-sided primary tumors of colorectal origin differ substantially in several aspects. Recent retrospective analyses show distinct efficacy of EGFR-epidermal growth factor receptor (EGFR)-directed therapies for left- and right-sided primary tumors. Current treatment guidelines have accommodated these findings such that for right-sided primary tumors, EGFR-directed therapy is no longer recommended. Instead, vascular endothelial growth factor (VEGF)-directed therapies are recommended frequently in first line, even in tumors with wild-type rat sarcoma (RAS) status. However, data supporting this recommendation are scarce. The purpose of this analysis was to investigate the efficacy of bevacizumab added to chemotherapy depending on the primary tumor localization in a retrospective setting. Patients and Methods: From the central clinical cancer registry of one of Germany's largest medical centers, data were analyzed for patients with metastatic colorectal cancer $(m C R C)$ treated with either chemotherapy alone (CT) or bevacizumab-containing regimens $(B E V / C T)$. Results: Of 1,080 documented $m C R C$ cases within the period of 2003 through 2016, 242 were treated with chemotherapy alone and 166 with bevacizumabcontaining regimes in any line of therapy meeting the criterion above. In patients with left-sided primary tumor localization, a significant survival benefit was found when bevacizumab was added to chemotherapy. Patients with right-sided primaries, instead, did not derive any advantage when bevacizumab was added to chemotherapy. For the whole group of patients, this translated into a trend towards
\end{abstract}

Correspondence to: Frank Jordan, Stenglinstr. 1, 86356 Augsburg, Germany. Tel: +49 821400161493, Fax: +49 821400172072, e-mail: Frank.Jordan@klinikum-augsburg.de

Key Words: Primary tumor location, bevacizumab, efficacy, chemotherapy, colorectal cancer. improved survival in bevacizumab-treated patients with $m C R C$. Conclusion: Adding bevacizumab to chemotherapy in $\mathrm{mCRC}$ may be beneficial only in patients with left-sided primary tumor, while those with right-sided primary tumors may have no additional benefit from the addition of bevacizumab. This hypothesis-generating analysis should provide a basis for in-depth analysis of this issue in future prospective trials.

Tumors from different regions of the colon are clinically and molecularly distinct (1-5). Right-and left-sided tumors differ substantially in several aspects. Right-sided tumors are more often found in female patients, have higher TNM staging at diagnosis, are often mucinous, have greater immunogenicity, more frequently exhibit microsatellite instability and have more activating mutations in $R A S$, proto-oncogene B-raf $(B R A F)$ and phosphatidylinositol 3-kinase catalytic subunit (PIK3CA) genes. Left-sided colorectal cancer, instead, has higher rates of chromosomal instability and more frequent gene expression profiles corresponding to epithelial growth factor receptor (EGFR) pathway activation (6-9). The tissue expression of vascular endothelial growth factor (VEGF)-A, the target of bevacizumab, has also been demonstrated to vary depending on the localization of the primary tumor, with higher expression in the distal colon and rectum than in the proximal colon (9). These different molecular characteristics translate into a worse prognosis of the group of patients with right-sided tumors.

Several analyses have suggested that sidedness may also be predictive of a treatment benefit from targeted therapies of the VEGF and EGFR pathways (10-20). For EGFRdirected therapies, the difference in efficacy according to sidedness is now widely accepted and further supported by a recent meta-analysis of several large prospective trials (14). Therefore, EGFR-directed antibodies as a combination partner with chemotherapy are no longer recommended in several national and international treatment guidelines for 
patients with right-sided primary tumor localization $(21,22)$. This has prompted the assumption that VEGF-directed therapies should be used instead. No predictive markers are clinically available for the selection of patients with metastatic colorectal cancer (mCRC) for treatment with bevacizumab. The published data on the predictive value of primary tumor localization for the efficacy of bevacizumab are inconclusive.

The purpose of this investigation was to explore the sidedependent efficacy of bevacizumab in addition to chemotherapy in colorectal cancer in a hypothesis-generating approach. We retrospectively analyzed data from a large clinical cancer registry in Germany for differences in the efficacy of bevacizumab in addition to chemotherapy.

\section{Patients and Methods}

Data source. The data reported here were extracted from the Augsburg Interdisciplinary Cancer Center Clinical Cancer Registry, which includes all cancer cases within the catchment area of Swabia (population size approximately 1.8 million) since 1998. Because of changes in the cancer registry electronic documentation system and the European Medicines Agency approval of bevacizumab in January 2005, our analysis was focused on 1,080 patients with mCRC treated at least once for any reason related to their malignant disease at Augsburg Medical Center, regardless of whether their actual cancer treatment took place there or in private medical oncology practices.

Definition of cohorts. The case documentation in the registry comprises gender, age at first diagnosis, date of death, the localization of the primary tumor according to ICD-10 (23), the first and sequential lines of systemic therapies, and surgery of metastases or other locally directed therapies.

Ontogenetically, left-sided colonic cancer derives from the embryonic hindgut, while right-sided colonic tumors derive from the embryonic midgut. The demarcation line is the distal third of the transverse colon. For practical reasons and like the majority of other clinical reports, our analysis uses the splenic flexure as the demarcation line. As the register documentation follows the ICD10 classification, tumors of the cecum, ascending and transverse colon were summarized as right-sided tumors (ICD-10 C180-184), whereas those of the splenic flexure, descending colon, sigmoid and rectum were recorded as left-sided primaries (ICD-10 C185, C186, C187, C190-250, C2091-C2096).

After restriction of all registered cases to unambiguously documented right-or left-sided colon or rectal cancer, and after exclusion of unclear localizations and double carcinomas, 943 cases remained. Cases not treated with chemotherapy or with only planned or unknown systemic therapy were excluded from the analysis. Three patients had to be excluded because there was a switch from bevacizumab to EGFR antibodies during first-line therapy; two other patients were excluded because they received additional imatinib in first line because of malignant comorbidities.

Two hundred and forty-two cases comprised the chemotherapyonly group (CT), in which all other targeted therapies, especially VEGF-and EGFR-directed therapies, were excluded.
Table I. Distribution according to primary tumor location and therapy.

\begin{tabular}{lccc}
\hline Therapy & \multicolumn{3}{c}{ Primary tumor location, n (\%) } \\
\cline { 2 - 4 } & Left colon & Right colon & Total \\
\hline BEV/CT & $115(28.2)$ & $51(12.5)$ & $166(40.7)$ \\
CT only & $174(42.7)$ & $68(16.7)$ & $242(59.3)$ \\
Total & $289(70.8)$ & $119(29.2)$ & $408(100.0)$ \\
\hline
\end{tabular}

BEV: Bevacizumab, CT: chemotherapy only.

The bevacizumab plus chemotherapy group (BEV/CT) consisted of 166 cases. In this group, bevacizumab was administered in any line of therapy. Patients who received EGFR-directed therapies and aflibercept were excluded from this cohort.

A separate cohort comprised 202 cases treated with cetuximab or panitumumab plus chemotherapy (EGFR/CT). In this cohort, EGFR-directed therapies were administered in any line of therapy and VEGF-directed therapies were not excluded.

Chemotherapy was not stratified according to the various potential regimens. Thus, first-line and further chemotherapy was any kind of FOLFOX (oxaliplatin, folinic acid, fluorouracil) or FOLFIRI (irinotecan, folinic acid, fluorouracil) regimen, but also FUFOX (oxaliplatin, flurorouracil), XELOX (oxaliplatin, capecitabine), CAPIRI (irinotecan, fluorouracil), capecitabine monotherapy and different fluorouracil/folinic acid regimens.

Some cases in the cohort had adjuvant chemotherapy, neoadjuvant radiochemotherapy, radiotherapy, interventional radiological procedures or surgery of metastases of liver or lung before, during or after initiation of first-line systemic therapy for metastatic disease. To minimize the bias from patients with oligometastatic disease, one cohort was established under exclusion of patients with resection of liver metastases.

The analyzed endpoints were median survival and probability of survival. Survival of individual cases was measured from the date of diagnosis of metastatic CRC to death from any reason.

Data analysis and statistics. The data was analyzed using SAS (SAS Institute GmbH, Heidelberg, Germany) and, in a second step, IBM SPSS Statistics (PASW 18; Armonk, NY, USA). The probability of survival was evaluated using Kaplan-Meier estimates of survival, and treatment groups were compared with the use of log-rank test using a 95\% confidence interval. $p$-Values of less than 0.05 were considered significant.

\section{Results}

In the period from 2003 to 2016, the registry included 424 patients with mCRC treated either with BEV/CT or CT. Of these, 166 patients $(40.7 \%)$ were treated with BEV/CT in any line of therapy, and 242 (59.3\%) comprised the CT group. For the entire treatment group, 289 patients $(70.8 \%)$ had left-sided primaries, whereas 119 patients $(29.2 \%)$ had right-sided primary tumor. Of the left-sided primaries, 115 (39.8\%) were treated with BEV/CT and $174(60.2 \%)$ were treated with CT only. Of the right-sided primaries, $51(42.9 \%)$ were treated 
Jordan et al: Primary Tumor Localization and Efficacy of Bevacizumab in CC

Table II. Distribution of the therapy-and primary tumor location according to age and gender.

\begin{tabular}{|c|c|c|c|c|c|}
\hline & \multicolumn{2}{|c|}{ Primary tumor localization } & \multicolumn{3}{|c|}{ Therapy } \\
\hline & Left colon $(n=289)$ & Right colon $(\mathrm{n}=119)$ & CT only $(n=242)$ & $\mathrm{BEV} / \mathrm{CT}(\mathrm{n}=166)$ & Total $(n=408)$ \\
\hline Median age (range), years & $66.6(27.9-86.9)$ & $68.9(33.0-86.9)$ & $68.9(27.9-86.9)$ & $65.0(30.2-85.5)$ & $67.7(27.9-86.9)$ \\
\hline $\operatorname{Men}(\%)$ & $173(59.9)$ & $60(50.4)$ & $138(57.0)$ & $95(57.2)$ & $233(57.1)$ \\
\hline Women $(\%)$ & $116(40.1)$ & $59(49.6)$ & $104(43.0)$ & $71(42.8)$ & $175(42.9)$ \\
\hline
\end{tabular}

BEV: Bevacizumab, CT: chemotherapy only.

Table III. Median overall survival (OS) for patients with left-and those with right-sided primaries according to therapy.

\begin{tabular}{lccccrr}
\hline & \multicolumn{2}{c}{ Left-sided primary } & \multicolumn{2}{c}{ Right-sided primary } & \multicolumn{2}{c}{ All patients } \\
\cline { 2 - 7 } OS & BEV/CT & CT & BEV/CT & CT & BEV/CT & CT \\
\hline $\begin{array}{l}\text { Median (months) } \\
\text { 95\% CI } \\
p \text {-Value }\end{array}$ & 31.51 & 18.42 & 21.09 & 18.59 & 27.34 & 18.42 \\
\hline
\end{tabular}

CI: Confidence interval.

with BEV/CT and $68(57.1 \%)$ were treated with CT only (Table I). The age distribution of the groups was well-balanced and we found few more men in all subgroups (Table II). For the entire group of BEV/CT and CT cases together, $21.3 \%$ of patients had resection of liver metastases, reflecting patients with limited disease. In the BEV/CT group, $25.3 \%$ of patients had resection of liver metastases compared to $18.6 \%$ in the CT-only group. The median follow-up of survivors within the entire cohort used for this study was 19.8 months.

In patients with left-sided primaries, there was a median overall survival of 31.5 months in the BEV/CT group compared with 18.4 months in the chemotherapy-only group. This translated into a significant overall survival advantage favoring bevacizumab-containing regimens in any line of therapy for patients with left-sided primary tumor $(p=0.034$; Figure 1A). In the cohort of right-sided primaries, the median survival was 21.1 months in the BEV/CT group and 18.6 months in the CT group (not significant upon log-rank testing; Figure 1B). For the entire group, there was a trend favoring bevacizumab-containing regimens which did not meet the level of statistical significance (Figure 1C). When patients with resection of liver metastases, i.e. oligometastatic cases, were excluded, the results remained almost the same except that the survival advantage for bevacizumab-containing regimens in the whole patient group became significant (Figure 2).

In summary, we found no survival benefit for the addition of bevacizumab to chemotherapy in patients with right-sided primary tumor, independently of whether or not they underwent resection of liver metastases. Patients with leftsided primaries, instead, did indeed have a benefit when bevacizumab was added to chemotherapy even when cases with resection of metastases were excluded. For the whole patient group, this translated into a non-significant trend towards improved overall survival in bevacizumab-treated patients with mCRC. This survival difference in the whole patient group became statistically significant when cases having undergone resection of metastases were excluded.

As an internal validation control of patients with CRC documented in our cancer registry, we also analyzed a group of 202 patients treated with EGFR-directed therapies (cetuximab or panitumumab) plus chemotherapy in any line of treatment to compare the results to published meta-analyses. VEGF-directed therapies were not excluded in this cohort. In the group with left-sided primary tumor, there was a significant advantage in the probability of survival for the EGFR/CT group over the CT group (Figure 3A). This translated into a significant survival advantage for EGFR/CT in the whole patient group (Figure 3C). In the 42 cases with right-sided primary tumor, however, treatment with EGFR/CT in any line resulted in a median survival of 27.9 months. There was no significant survival advantage over CT only when EGFR-directed therapies were used in patients with right-sided primary tumor (Figure 3B).

Taken together, neither the addition of bevacizumab, nor the addition of EGFR-directed therapies to chemotherapy had a significant impact on survival in patients with rightsided primary tumor. 

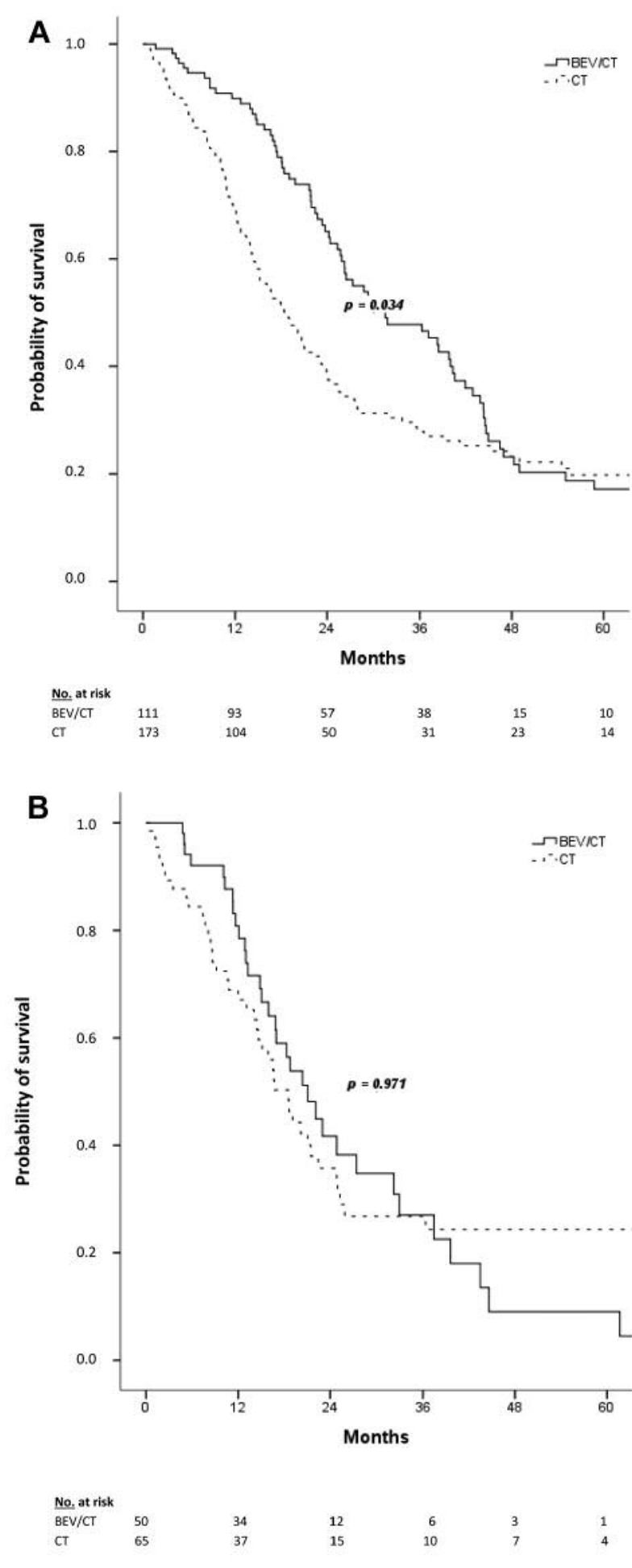

\section{Discussion}

Recent studies demonstrated a prognostic and predictive relevance of primary tumor localization in $\mathrm{mCRC}$ and a markedly greater benefit from EGFR-directed therapies in

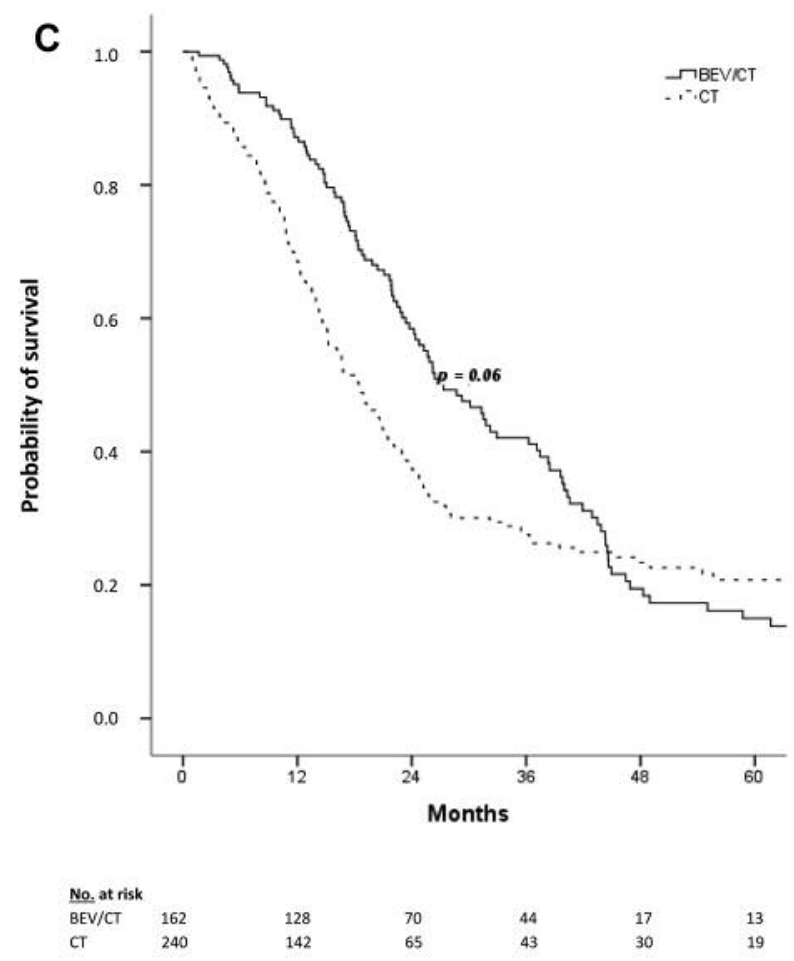

Figure 1. Kaplan-Meier curves of probability of overall survival according to treatment with bevacizumab (BEV) or without (chemotherapy-only; CT) for patients with A: left-sided primary, B: right-sided primary, and C: all patients.

patients with $R A S$ wild-type left-sided primaries compared to right-sided ones $(14,17,20)$. Several current treatment guidelines have accommodated these results $(21,22)$. For right-sided primary tumors, EGFR-directed therapy in first line is no longer recommended. Instead, VEGF-directed therapies are commonly preferred in first-line and further lines even in those with $R A S$ wild-type tumors if the primary tumor is right-sided $(21,22)$. An additional effect on overall survival of anti-VEGF therapy over treatment with chemotherapy alone is widely accepted but has not been demonstrated unambiguously and published data concerning this issue are scarce.

The purpose of our analysis was to investigate the efficacy of bevacizumab added to chemotherapy in relation to the primary tumor localization in a non-selected population and real-life setting outside of clinical trials from a database of a cancer registry. In fact, we found no survival benefit upon adding bevacizumab to chemotherapy in patients with rightsided primary tumor. As expected, patients with left-sided primaries did have a substantial benefit when bevacizumab was added to chemotherapy. This translated into a nonsignificant trend towards a better survival considering the entire group of left-and right-sided primaries together. This 

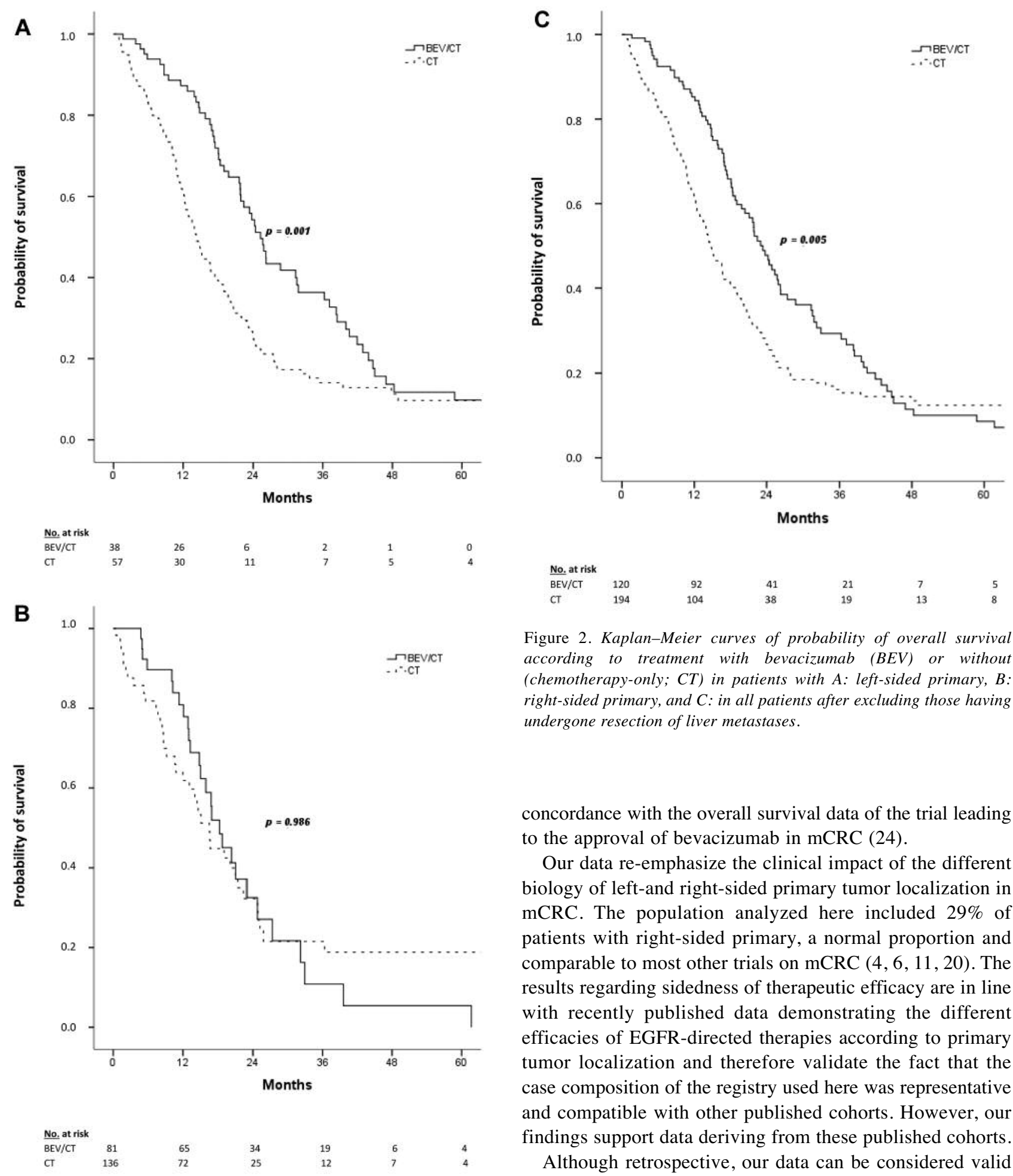

Figure 2. Kaplan-Meier curves of probability of overall survival according to treatment with bevacizumab (BEV) or without (chemotherapy-only; $C T$ ) in patients with A: left-sided primary, B: right-sided primary, and $C:$ in all patients after excluding those having undergone resection of liver metastases.

concordance with the overall survival data of the trial leading to the approval of bevacizumab in mCRC (24).

Our data re-emphasize the clinical impact of the different biology of left-and right-sided primary tumor localization in mCRC. The population analyzed here included $29 \%$ of patients with right-sided primary, a normal proportion and comparable to most other trials on $\operatorname{mCRC}(4,6,11,20)$. The results regarding sidedness of therapeutic efficacy are in line with recently published data demonstrating the different efficacies of EGFR-directed therapies according to primary tumor localization and therefore validate the fact that the case composition of the registry used here was representative and compatible with other published cohorts. However, our findings support data deriving from these published cohorts.

Although retrospective, our data can be considered valid especially because confounders such as EGFR-directed or other targeted therapies were excluded in the BEV/CT and $\mathrm{CT}$ cohorts and the analysis was not restricted to first-line application of bevacizumab. This issue has not been addressed in other published work investigating the prognostic and predictive value of primary tumor localization 

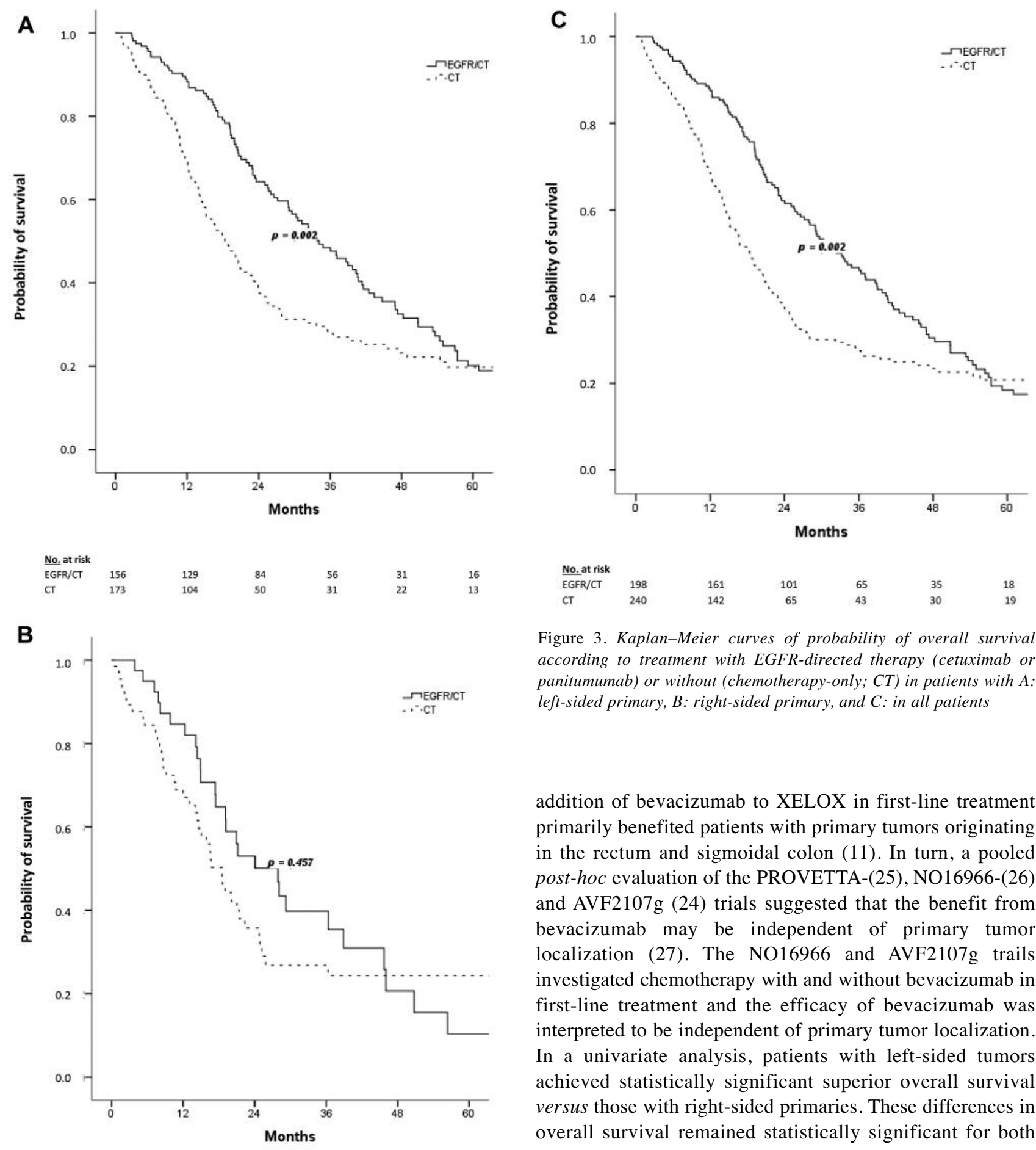

Figure 3. Kaplan-Meier curves of probability of overall survival according to treatment with EGFR-directed therapy (cetuximab or panitumumab) or without (chemotherapy-only; CT) in patients with A: left-sided primary, B: right-sided primary, and $C:$ in all patients

addition of bevacizumab to XELOX in first-line treatment primarily benefited patients with primary tumors originating in the rectum and sigmoidal colon (11). In turn, a pooled post-hoc evaluation of the PROVETTA-(25), NO16966-(26) and AVF2107g (24) trials suggested that the benefit from bevacizumab may be independent of primary tumor localization (27). The NO16966 and AVF2107g trails investigated chemotherapy with and without bevacizumab in first-line treatment and the efficacy of bevacizumab was interpreted to be independent of primary tumor localization. In a univariate analysis, patients with left-sided tumors achieved statistically significant superior overall survival versus those with right-sided primaries. These differences in overall survival remained statistically significant for both subgroups, bevacizumab plus chemotherapy and chemotherapy-only, respectively. Multivariable analyses of the NO16966 and AVF2107g trials also showed that primary tumor localization and the use of bevacizumab were independent prognostic factors for overall survival. The conclusion of the authors, that the efficacy of bevacizumab was independent of primary tumor localization is not easy to interpret, especially because the subgroups of left-and right addressed the efficacy of bevacizumab according to primary tumor localization. In 2013, Boisen et al. reported that the 
sided primaries were not stratified for bevacizumab with/without chemotherapy $(26,27)$.

The AGITG MAX trial compared first-line capecitabine with capecitabine plus bevacizumab versus capecitabine plus bevacizumab plus mitomycin $\mathrm{C}$ in advanced CRC. A posthoc analysis of this trial suggested a benefit from bevacizumab in addition to chemotherapy independently of primary tumor localization regarding the reported endpoint progression-free survival (28). Similar results were reported in a prospective trial on a Chinese cohort (29).

In contrast, our study evaluated median overall survival and bevacizumab was added to chemotherapy in any line, not only first-line. Thus, the role of bevacizumab in mCRC deriving from right-or left-sided primaries is still somewhat ambiguous. Nevertheless, our study may be viewed as an additional reason towards critically discussing the general use of bevacizumab in mCRC independently of primary tumor localization. Of course, our study was retrospective and therefore exploratory and hypothesis-generating in nature. In addition, our patient population differs from those of other studies because it analyzed an 'all-comers' population without inclusion, or exclusion criteria before entering the data-pool as is the case in prospective clinical trials. Patients with central nervous system metastases, patients having undergone radiotherapy, or interventions such as elective internal radiation therapy and transarterial chemoembolisation, were not excluded. Our data show patients with very long survival. Over $50 \%$ of the patients surviving more than 45 months had undergone resection of liver metastases. In comparison, only $21 \%$ of the entire population had undergone resection of metastases, suggesting that the long-term survivors are oligo-metastatic cases. Both cohorts with or without resection of liver metastases had similar outcomes, meaning there was no survival advantage of adding bevacizumab to chemotherapy in those with $\mathrm{mCRC}$ when the primary was right-sided.

The cancer registry used for this analysis does not provide information on the performance status, comorbidities or genetic alterations such as $R A S$ and $B R A F$ mutational status or microsatellite instability. The chemotherapeutic regimen and the sequence of therapy in our cohort was not predefined, in contrast to the trials that were used for the cited metaanalysis (17). In those trials, the chemotherapy backbone was FOLFOX or FOLFIRI, whereas in our cohort additional regimens such as FUFOX, CAPOX, CAPIRI, capecitabine, different 5-FU monotherapies were also used in some patients.

Taken together, our data suggest that adding bevacizumab to chemotherapy in mCRC in a real-world-setting might only be beneficial in patients with left-sided primary tumors, while patients with right-sided primaries may have no additional benefit from inclusion of bevacizumab in systemic treatments. Thus, in addition to the now generally accepted lack of effect of EGFR-targeted therapies in patients with right-sided primary tumor, our data suggest the same for bevacizumab. This could question current guideline recommendations as data such as the those presented here suggest that right-sided primary tumor localization might be a negative predictive factor for the use of either of the monoclonal antibodies currently approved for mCRC. We suggest further investigation of this issue in future prospective clinical trials.

\section{Conflicts of Interests}

Dr. Frank Jordan declares to have no financial, personal or other relationships with other people or organizations within that could inappropriately influence his work, and, in regard to bevacizumab or other therapies used in CRC, declares he has no employment, no consultancies, stock ownership, honoraria, paid expert testimony, patent applications/registrations and grants and any other funding. He has received traveling expenses by Amgen, Astra Zeneca, Bayer, BMS, Boehringer-Ingelheim, Celgene, GSK, Ipsen, Janssen, Lilly, Merck, MSD, Novartis, Pfizer, Roche, Servier, Sanofi.

\section{References}

1 Missiaglia E, Jacobs B, D'Ario G, Di Narzo AF, Soneson C, Budinska E, Popovici V, Vecchione L, Gerster S, Yan P, Roth AD, Klingbiel D, Bosman FT, Delorenzi M and Tejpar S: Distal and proximal colon cancers differ in terms of molecular, pathological, and clinical features. Ann Oncol 25(10): 19952001, 2014.

2 Yamauchi M, Lochhead P, Morikawa T, Huttenhower C, Chan AT, Giovannucci E, Fuchs C and Ogino S: Colorectal cancer: A tale of two sides or a continuum? Gut 61(6): 794-797, 2012.

3 Yamauchi M, Morikawa T, Kuchiba A, Imamura Y, Qian ZR, Nishihara R, Liao X, Waldron L, Hoshida Y, Huttenhower C, Chan AT, Giovannucci E, Fuchs C and Ogino S: Assessment of colorectal cancer molecular features along bowel subsites challenges the conception of distinct dichotomy of proximal versus distal colorectum. Gut 61(6): 847-854, 2012.

4 Carethers JM: One colon lumen but two organs. Gastroenterology 141(2): 411-412, 2011.

5 Bufill JA: Colorectal cancer: Evidence for distinct genetic categories based on proximal or distal tumor location. Ann Intern Med 113(10): 779-788, 1990.

6 LaPointe LC, Dunne R, Brown GS, Worthley DL, Molloy PL, Wattchow D and Young GP: Map of differential transcript expression in the normal human large intestine. Physiol Genomics 33(1): 50-64, 2008.

7 Iacopetta B: Are there two sides to colorectal cancer? Int J Cancer 101(5): 403-408, 2002.

8 Guinney J, Dienstmann R, Wang X, de Reynies A, Schlicker A, Soneson C, Marisa L, Roepman P, Nyamundanda G, Angelino P, Bot BM, Morris JS, Simon IM, Gerster S, Fessler E, De Sousa EMF, Missiaglia E, Ramay H, Barras D, Homicsko K, Maru D, Manyam GC, Broom B, Boige V, Perez-Villamil B, Laderas T, Salazar R, Gray JW, Hanahan D, Tabernero J, Bernards R, Friend SH, Laurent-Puig P, Medema JP, Sadanandam A, Wessels L, Delorenzi M, Kopetz S, Vermeulen L and Tejpar S: The consensus molecular subtypes of colorectal cancer. Nat Med 21(11): 1350-1356, 2015. 
9 Bendardaf R, Buhmeida A, Hilska M, Laato M, Syrjanen S, Syrjanen K, Collan Y and Pyrhonen S: Vegf-1 expression in colorectal cancer is associated with disease localization, stage, and long-term disease-specific survival. Anticancer Res 28(6B): 3865-3870, 2008.

10 Benedix F, Kube R, Meyer F, Schmidt U, Gastinger I, Lippert H and Colon/Rectum Carcinomas Study G: Comparison of 17,641 patients with right- and left-sided colon cancer: Differences in epidemiology, perioperative course, histology, and survival. Dis Colon Rectum 53(1): 57-64, 2010.

11 Boisen MK, Johansen JS, Dehlendorff C, Larsen JS, Osterlind K, Hansen J, Nielsen SE, Pfeiffer P, Tarpgaard LS, Hollander NH, Keldsen N, Hansen TF, Jensen BB and Jensen BV: Primary tumor location and bevacizumab effectiveness in patients with metastatic colorectal cancer. Ann Oncol 24(10): 2554-2559, 2013.

12 Modest DP, Schulz C, von Weikersthal LF, Quietzsch D, von Einem JC, Schalhorn A, Vehling-Kaiser U, Laubender RP, Giessen C, Stintzing S and Heinemann V: Outcome of patients with metastatic colorectal cancer depends on the primary tumor site (midgut $v s$. Hindgut): Analysis of the fire1-trial (fufiri or mirox as first-line treatment). Anticancer Drugs 25(2): 212-218, 2014.

13 von Einem JC, Heinemann V, von Weikersthal LF, VehlingKaiser U, Stauch M, Hass HG, Decker T, Klein S, Held S, Jung A, Kirchner T, Haas M, Holch J, Michl M, Aubele P, Boeck S, Schulz C, Giessen C, Stintzing S and Modest DP: Left-sided primary tumors are associated with favorable prognosis in patients with kras codon 12/13 wild-type metastatic colorectal cancer treated with cetuximab plus chemotherapy: An analysis of the aio krk-0104 trial. J Cancer Res Clin Oncol 140(9): 16071614, 2014.

14 Holch JW, Ricard I, Stintzing S, Modest DP and Heinemann V: The relevance of primary tumour location in patients with metastatic colorectal cancer: A meta-analysis of first-line clinical trials. Eur J Cancer 70: 87-98, 2017.

15 Goldstein DA, Chen Q, Ayer T, Chan KKW, Virik K, Hammerman A, Brenner B, Flowers CR and Hall PS: Bevacizumab for metastatic colorectal cancer: A global costeffectiveness analysis. Oncologist 22(6): 694-699, 2017.

16 Ilic I, Jankovic S and Ilic M: Bevacizumab combined with chemotherapy improves survival for patients with metastatic colorectal cancer: Evidence from meta analysis. PLoS One 11(8): e0161912, 2016.

17 Tejpar S, Stintzing S, Ciardiello F, Tabernero J, Van Cutsem E, Beier F, Esser R, Lenz HJ and Heinemann V: Prognostic and predictive relevance of primary tumor location in patients with ras wild-type metastatic colorectal cancer: Retrospective analyses of the crystal and fire-3 trials. JAMA Oncol, 2016. doi: 10.1001/jamaoncol.2016.3797. [Epub ahead of print]

18 Loupakis F, Yang D, Yau L, Feng S, Cremolini C, Zhang W, Maus MK, Antoniotti C, Langer C, Scherer SJ, Muller T, Hurwitz HI, Saltz L, Falcone A and Lenz HJ: Primary tumor location as a prognostic factor in metastatic colorectal cancer. J Natl Cancer Inst 107(3), 2015. doi: 10.1093/jnci/dju427
19 Brule SY, Jonker DJ, Karapetis CS, O'Callaghan CJ, Moore MJ, Wong R, Tebbutt NC, Underhill C, Yip D, Zalcberg JR, Tu D and Goodwin RA: Location of colon cancer (right-sided versus left-sided) as a prognostic factor and a predictor of benefit from cetuximab in ncic co.17. Eur J Cancer 51(11): 1405-1414, 2015.

20 Arnold D, Lueza B, Douillard JY, Peeters M, Lenz HJ, Venook A, Heinemann V, Van Cutsem E, Pignon JP, Tabernero J, Cervantes A and Ciardiello F: Prognostic and predictive value of primary tumour side in patients with ras wild-type metastatic colorectal cancer treated with chemotherapy and egfr directed antibodies in six randomized trials. Ann Oncol 28(8): 17131729, 2017.

21 Network NCC. NCCN Guidelines version 1.2018 on colon cancer, 2018.

22 Arnold D, Seufferlein T and Krebsgesellschaft AIOdD: Statement der AIO-KRK-Leitgruppe zur Wahl der Erstlinientherapie bei Patienten mit metastasiertem Kolorektalkarzinom ohne Nachweis einer RAS Mutation unter Berücksichtigung der primären Tumorlokalisation, 2016.

23 ICD-10, International Statistical Classification of Diseases and Related Health Problems, No. 10, Version 2016.

24 Hurwitz H, Fehrenbacher L, Novotny W, Cartwright T, Hainsworth J, Heim W, Berlin J, Baron A, Griffing S, Holmgren E, Ferrara N, Fyfe G, Rogers B, Ross R and Kabbinavar F: Bevacizumab plus irinotecan, fluorouracil, and leucovorin for metastatic colorectal cancer. N Engl J Med 350(23): 2335-2342, 2004.

25 NIH National Library of Science ctg. Evaluation of VEGF polymorphism as perdictive factor in metastatic colorectal cancer treated with FOLFIRI plus bevacizumab (Provetta), 2017.

26 Saltz LB, Clarke S, Diaz-Rubio E, Scheithauer W, Figer A, Wong R, Koski S, Lichinitser M, Yang TS, Rivera F, Couture F, Sirzen $\mathrm{F}$ and Cassidy J: Bevacizumab in combination with oxaliplatin-based chemotherapy as first-line therapy in metastatic colorectal cancer: A randomized phase iii study. J Clin Oncol 26(12): 2013-2019, 2008.

27 Cassidy J, Clarke S, Diaz-Rubio E, Scheithauer W, Figer A, Wong R, Koski S, Rittweger K, Gilberg F and Saltz L: Xelox $v s$. folfox-4 as first-line therapy for metastatic colorectal cancer: No16966 updated results. Br J Cancer 105(1): 58-64, 2011.

28 Price TJ, Buizen L, Hardingham J, Lee CK, Townsend A, Bruhn M, Simes RJ, Wilson K, Gebski V and Tebbutt NC: Molecular subgroups from the agitg max trial; right or left primary site of colorectal cancer and outcomes for metastatic colorectal cancer (mcrc). Annals of Oncology 25( Suppl 4): iv167-iv209, 2014.

29 Sun DC, Shi Y, Wang YR, Lv Y, Yan H, Mao H, Wang ZK, Wu ZY, Shi WW and Dai GH: Kras mutation and primary tumor location do not affect efficacy of bevacizumab-containing chemotherapy in stagae iv colorectal cancer patients. Sci Rep 7(1): 14368, 2017. 